\title{
The Influence of Voluntary Upper Body Exercise on the Performance of Stimulated Paralysed Human Quadriceps
}

\author{
Henry M. Franken, Peter H. Veltink, Maarten J. IJzerman, Frank S. Withaar and Herman \\ B. K. Boom
}

Institute for Biomedical Technology/Faculty of Electrical Engineering, University of Twente, Enschede, The Netherlands

\begin{abstract}
Summary: In this study the influence of voluntary upper body exercise on the performance of stimulated paralysed human quadriceps was investigated in five subjects with spinal cord lesions in the thoracic spine. The experimental setup consisted of computer-controlled stimulation of the quadriceps using electrodes on the surface of the skin, a dynamometer for isometric or isokinetic loading of the lower leg, and a rowing ergometer for upper body exercise. In all subjects, quadriceps fatigue tests were conducted to study the influence of upper body exercise on knee torque during sustained continuous or intermittent stimulation of quadriceps. The relative asymptotic torque appeared to be significantly higher with the presence of upper body exercise than without. This was consistently found both between trials (starting with or without upper body exercise) as well as within trials, when upper body exercise was started or stopped during the trial. No significant influence of upper body exercise on the time constant of initial torque decline was found. (c) 1997 Elsevier Science Ltd. All rights reserved.
\end{abstract}

Key Words: Quadriceps muscles-Functional electrical stimulation-Paraplegia-Upper body exercise-Muscle performance and fatigue.

J. Electromyogr. Kinesiol., Vol. 7, 67-77, March

\section{INTRODUCTION}

The enhancement of locomotor functions in spinal cord injured (SCI) patients using functional neuromuscular stimulation (FNS) is hampered by many problems ${ }^{1,15,24,26,27,31,36}$. One of these is muscle fatigue, which limits the application of FNS. Muscle

Received 11 March 1995.

Revised 11 January 1996.

Accepted 4 February 1996.

Correspondence and reprint requests to P. H. Veltink, Biomedical Engineering Division, Faculty of Electrical Engineering, University of Twente, P.O. Box 217, 7500 AE Enschede, The Netherlands. Tel: +31-53-4892765; Fax: +31-53-4892287; E-mail: p.h.veltink@el.utwente.nl. force typically decays exponentially in time, reaching relatively low asymptotic levels $s^{6,12,13,28}$. This time-variant muscle behaviour compliçates the control of FNS in restoring a certain locomotor function. If the asymptotic force level is too low for a certain task, the time in which this task can be performed is also limited ${ }^{14,30,32}$

Several aspects of output and fatigue of paralysed human quadriceps have been studied without considcring the influence of upper body activity. It has been well documented ${ }^{34}$ that muscle conditioning by long-term electrical stimulation can markedly improve the performance and fatigue resistance of stimulated paralysed muscle. Caroll et al. ${ }^{7}$ showed 
that higher frequencies of stimulation result in faster and relatively larger decay of quadriceps output. Franken et al. ${ }^{12}$ reported that the number of pulses in a given stimulation burst was a larger determinant of fatiguc in paralysed quadriceps than the duty cycle during sustained intermittent (surface) stimulation. In accordance with this finding, Boom et al. ${ }^{6}$ showed that intermittent (surface) stimulation exhibited a significantly lower rate of torque decline compared to continuous stimulation. Moreover, the torque decline rate and relative asymptotic torque appeared independent of recruitment level ${ }^{6}$. This may be explained by random (not orderly) recruitment of motor units when using surface stimulation. Baratta et al. ${ }^{3}$ showed a dependence of torque decline rate on recruitment level in cat soleus muscle, when motor units were recruited in an orderly manner according to the size principle using a tripolar nerve cuff electrode. Variation of the interpulse interval within a stimulation burst, specifically the application of doublets and triplets, has also been reported to augment muscle output and reduce fatigue $^{4,5,13,19,20,25,34,35,39}$. Furthermore, in the case of isokinetic concentric contractions of paralysed quadriceps, it has been shown that smaller contraction velocities reduced muscle fatigue ${ }^{12}$. However, the rate and relative decay of quadriceps force were not significantly dependent on muscle length ${ }^{16.28}$.

In the above studies the subjects were all seated and performed no significant upper body activity. However, it is a common observation that FNSsupported paraplegic walking is accompanied by significant upper body activity $15,24,29,36$. The influence of upper body activity on the performance of stimulated lower extremity muscles has not extensively been addressed. Glaser et al. ${ }^{17}$ and Glaser ${ }^{18}$ reported on hybrid physical exercise of high-level SCI subjects using the combination of quadriceps stimulation in both legs with voluntary arm crank ergonometer exercise. They concluded that the effect of physical training in terms of oxygen uptake, pulmonary ventilation and heart rate increased using the above hybrid method in comparison to either training method by itself. They also reported a higher quadriceps endurance during 20 stimulation cycles (total duration of $3.5 \mathrm{~min}$ ) and simultaneous upper body exercise in comparison to quadriceps stimulation only.

In healthy people, the regulation of exercise starts with a basic pattern of cardiovascular activity. This central command consists of a vagal withdrawal and increased sympathetic activity ${ }^{2,30,33}$. The cardio- vascular activity during exercise, increased heart rate and pressure is modulated by chemoreflexes, which are triggered by muscle metabolites or by a baroreflex, which are in turn triggered by low blood pressure $^{33}$. The key in the regulation of blood flow during exercise is the redistribution of the cardiac output. Cholinergic sympathetic innervation causes vasodilatation in skeletal muscles, thereby increasing their share of the cardiac output. Furthermore, the activity of sympathetic adrenergic vasoconstrictor fibres reduces the blood flow to the abdominal organs and the skin $^{2}$.

In SCI patients there may be four causes for a deficient local blood flow in the muscles: (a) the regulation of blood flow by means of peripheral reflexes may have disappeared - if this is the case, FNS therapy may not lead to increased circulation; (b) sympathetic control may be lust in high-level lesions (T1-T6), and as a consequence the cardiovascular performance may only be increased by vagal withdrawal; (c) the paralysis of leg muscles leads to a decreased venous return of the blood because the pump activity is lacking, which affects cardiac output ${ }^{21-23}$; and (d) the autonomic innervation may be deficient. This may result in a less efficient redistribution of the cardiac output ${ }^{21-23}$.

Combined upper and lower extremity exercise in SCI patients can counteract two of the above-mentioned causes for a deficient local blood flow, those being peripheral reflex control and increased venous blood return by muscle activity. The peripheral reflex control of the legs can possibly be bypassed through upper body exercise. This hypothesis is supported by the study of Hopman et al. ${ }^{21,23}$. They found an increase in blood flow through the femoral artery during upper body exercise. Because femoral artery blood flow increases during upper body exercise, it is expected that the performance during lower extremity FNS will increase ${ }^{17,18}$. Furthermore, because FNS enhances the muscle pump mechanism, cardiac output will be increased, thus influencing the performance positively ${ }^{8.9}$. The increased cardiac output can be used partly for lower extremity exercise.

The objective of the present study is to investigate experimentally the influence of voluntary upper body activity (rowing) on the performance and fatigue of stimulated quadriceps in paraplegics. In correspondence with our previous research on muscle fatigue $^{6,12.13}$, we were particularly interested in the influence of upper body exercise on the decay time constant and the asymptotic level of the muscle 
output. We examined the quadriceps muscles because they play an important role in paraplegic locomotion.

\section{METHODS}

\section{Subjects}

Five SCI subjects with lesions in the thoracic region participated in this study (Table 1). The enthusiasm of the subjects with respect to the current study was considered important because they had to comply with large physical efforts. We examined the left quadriceps in all subjects, which appeared normally excitable and without significant occurrence of spasm. All subjects had performed an FNS home training programme prior to the experiment for different individual periods (Table 1). The subjects were asked to train for half-an-hour each day. The training programme consisted of cyclical quadriceps stimulation ( $2 \mathrm{~s}$ on/ $2 \mathrm{~s}$ off) in both legs in a sitting position with the lower legs loaded with a $5 \mathrm{~kg}$ weight.

\section{Experimental Set-Up}

\section{Dynamometer}

We used a KINCOM 125ES (Kinetic Communicator Exercise System) dynamometer bench (Chattecx Corporation, Chattanooga Group, Chattanooga, TN, U.S.A.) to load the lower leg isometrically or isokinetically and to measure knee angle, velocity and torque (Figure 1). The left lower leg was attached to the rotating arm just above the ankle. Straps fixed the hip angles at approximately $100^{\circ}$. Additional straps around the knee and shoulder ensured a static body position during upper body exercise. In Franken et al. ${ }^{12}$ the current dynamometer set-up is described in detail.

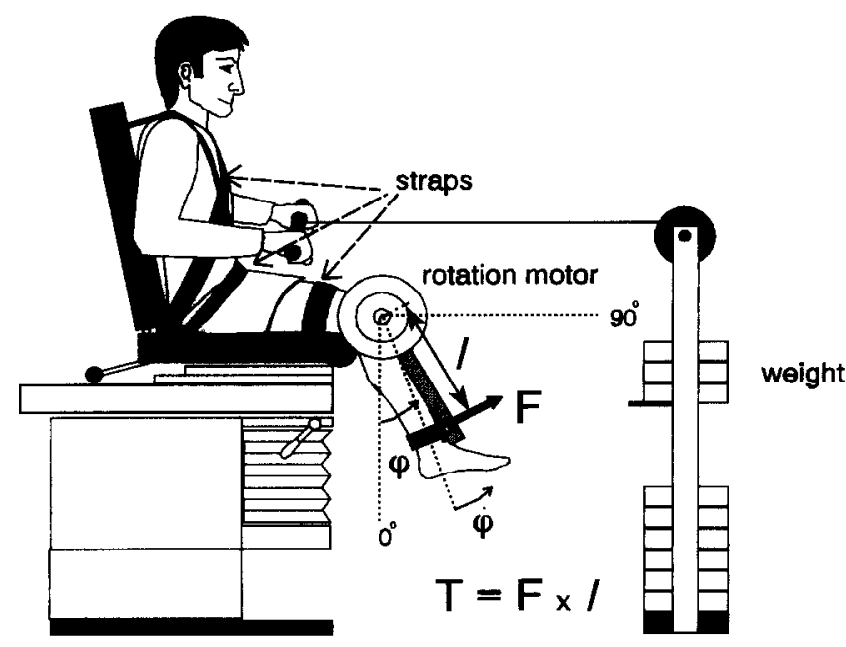

FIG. 1. The experimental set-up. The angular position $\varphi$, velocity $\dot{\varphi}$, and joint torque $T$ are defined in the figure. Knee angle and velocity were measured at the motor axis. Joint force $F$ perpendicular to the lever arm was measured just above the ankle with a force sensor on the lever arm. Joint torque $T$ resulted from multiplication of joint force $F$ with the moment arm I. The patient was strapped at the shoulder, hip and above the knee to measure knee torque only and to ensure static position of the body during upper body exercise.

\section{Voluntary Upper Body Exercise}

Upper body exercise consisted of rowing at a frequency of $1 \mathrm{~Hz}$. The subjects were instructed to perform a full-span rowing motion. Rowing intensity could be varied by adjusting the weight that had to be pulled up via a pulley (Figure 1). The weight was set between $10-15 \%$ of the maximal weight the subject could hold for several seconds in the experimental set-up, which was tested at the start of the experiment using a weigh-beam.

\section{Stimulation and Data Recording}

Using two adhesive surface electrodes (Pals, Axelgaard Manufacturing Co. Ltd, Fallbrook, CA,

TABLE 1. Information about the five participating paraplegic subjects

\begin{tabular}{lccccc}
\hline Subject & Sex & Year of birth & Year of injury & Training & Level of injury \\
\hline A & male & 1974 & 1989 & 36 months & T5-6 \\
B & male & 1966 & 1979 & 2 months & T11-12 \\
C & male & 1950 & 1990 & 26 months & T9-10 \\
D & male & 1944 & 1983 & 3 months & T9 \\
E & male & 1965 & 1988 & 4 months & T4 \\
\cline { 2 - 5 }
\end{tabular}

All subjects had a complete spinal cord lesion in the thoracic region. The quadriceps in their left leg appeared normally excitable without significant occurrence of spasm. The period of consequent training with FNS prior to the experiment is also given. 
U.S.A.; $5 \times 9 \mathrm{~cm}$ ), the left quadriceps were stimulated by placing the cathode over the motor points of rectus femoris/vastus lateralis and the anode over the motor point of vastus medialis. This electrode placement was also used during the FNS home training session. An IBM-AT compatible computer with A/D facilities (Analog Devices, RTI-815, 12bit) sampled the data at a rate of $100 \mathrm{~Hz}$ and controlled a current stimulator that generated monophasic rectangular pulses. A/D conversion and stimulus pulse generation were synchronously controlled under interrupt. The adopted nomenclature for stimulation parameters is explained in Figure 2. During the experiment, pulse width $(300-500 \mu \mathrm{s})$ and pulse amplitude $(0-100 \mathrm{~mA})$ were fixed to obtain a stable submaximal recruitment of quadriceps muscles. Recruitment curves indicated that knee torque varied relatively little with stimulation amplitude when using these stimulation parameters, although maximal recruitment was not reached in all cases. The interpulse interval was fixed at $20 \mathrm{~ms}$. Such a relatively small interpulse interval may be required to produce sufficient impulse during short contractions of muscles in FNS-supported gait when using external skin electrodes. The parameter settings correspond to those in previous fatigue studies $^{12,13}$. Knee angle, velocity and torque were sampled at $100 \mathrm{~Hz}$. Heart rate was monitored by a photoelectric pulse sensor with earclip (Kettler ${ }^{\mathrm{TM}}$ ). The displayed heart rate and time were videotaped together as the subject performed the trial. These data samples, heart rate registration, and the stimulus data were stored for off-line analysis. The measurement system was calibrated prior to each measurement day.

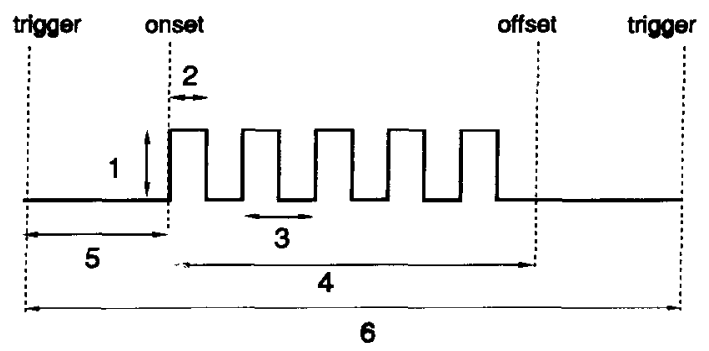

FIG. 2. The stimulation nomenclature. 1=pulse amplitude, 2=pulse width, 3=interpulse interval, 4=burst duration, 5=wait time, $6=$ cycle time. The duty cycle equals to the burst duration divided by the cycle time. The number of pulses equals to the burst duration divided by the interpulse interval. The wait time is the time between a trigger to contract and the onset of the stimulation burst. The duration of the stimulation burst determines the offset of the stimulation.

\section{Protocol}

Six fatigue trials were performed on one day. Each trial lasted $10 \mathrm{~min}$ followed by $50 \mathrm{~min}$ of rest. In each trial upper body activity was performed either at the beginning ( 2 min before and until 6 min after the start of stimulation) or during the last $4 \mathrm{~min}$ of stimulation ( $6 \mathrm{~min}$ after the start of stimulation and until the end of the trial). Stimulation was performed either continuously (trials 1 and 2) or intermittently (trials 3 to 6). Loading was either isometric (trials 1 to 4 ) or isokinetic (trials 5 and 6). This is summarized in Table 2.

The quadriceps was warmed up prior to trial 1 to make the initial state of the quadriceps in this trial comparable to the following trials. This warmup session consisted of 5 min of low-level intermittent isometric stimulation followed by $20 \mathrm{~min}$ of rest. Immediately after the warm-up session an isometric recruitment curve was measured at a knee angle of $50^{\circ}$ in order to select a stimulation pulse amplitude for a stable (sub)maximal recruitment well above the steep part of the recruitment curve. An isometric recruitment curve was again measured after trial 6 to check that the recruitment was still at the same stable (sub)maximal level. Prior to the commencement of each fatigue trial, the quadriceps muscle was warmed up by passive joint movements at $40^{\circ} \mathrm{s}^{-1}$ for approximately $1 \mathrm{~min}$. This avoided initial spasm at the beginning of the trials and resulted in a repeatable passive torque response in the absence of stimulation before the beginning of the isokinetic trials 5 and 6 . This was required for the estimation of the torque actively generated by the stimulated quadriceps during these trials.

\section{Off-Line Data Analysis}

\section{Torque-Time Integral}

The torque actively generated by the stimulated quadriceps muscles was computed by subtracting the passive knee torque due to gravity and the passive characteristics of the knee and $\operatorname{shank}^{12}$. In the case of the isometric trials 1 to 4 , this was done by subtracting the constant torque level prior to quadriceps stimulation from the torque measured during stimulation. In the isokinetic trials 5 and 6 , the average torque registration of 10 passive cycles prior to the commencement of the trial was subtracted from the torque registration of each cycle of the trial. Integration of the active torque per cycle 
TABLE 2. Experiment parameter settings

\begin{tabular}{lccccc}
\hline Trial & Loading & $\begin{array}{c}\text { Velocity } \\
\left(\mathrm{deg} \mathrm{s}^{-1}\right)\end{array}$ & $\begin{array}{c}\text { Angle (range) } \\
(\mathrm{deg})\end{array}$ & $\begin{array}{c}\text { Duty cycle } \\
(\%)\end{array}$ & $\begin{array}{c}\text { Upper body activity } \\
(\mathrm{min})\end{array}$ \\
\hline 1 & Isometric & 0 & 50 & 100 & $6-10$ \\
2 & Isometric & 0 & 50 & 100 & $-2-6$ \\
3 & Isometric & 0 & 50 & 32 & $6-10$ \\
4 & Isometric & 0 & 50 & 32 & $-2-6$ \\
5 & Isokinetic & 40 & $32-68$ & 32 & $6-10$ \\
6 & Isokinetic & 40 & $32-68$ & $2-6$ \\
\hline
\end{tabular}

All trials lasted for $10 \mathrm{~min}$. All cycle times of the intermittent stimulation (trials 3 to 6 ) and the isokinetic triangular movements (trials 5 and 6 ) were set to $2 \mathrm{~s}$. In the isokinetic trials 5 and 6 , stimulation started at a knee angle of $35^{\circ}$ in the concentric phase of the movement.

of $2 \mathrm{~s}$ yielded the torque-time integral (one sample per cycle). Continuous stimulation in trials 1 and 2 was treated as intermittent stimulation with a duty cycle of $100 \%$ and a cycle time of $2 \mathrm{~s}$ in order to allow comparison with the other trials. The decay in torque-time integral in the first $6 \mathrm{~min}$ of each trial was parameterized by fitting:

$$
\hat{M}(t)=M_{\max } \cdot[(1-\xi) \cdot \exp (-t / \tau)+\xi]
$$

where $\hat{M}$ is the estimated torque-time integral; $t$ is time; $M_{\max }$ is the initial maximum in the torquetime integral; $\tau$ is a time constant indicating the rate of fatigue development; and $\xi$ the relative asymptote to which $M(t)$ descends ${ }^{6,12,13}$. It should be noted that this asymptote is a relative value with respect to $M_{\max }$. The RMS fitting error was small ( $<2 \%$ of $\left.M_{\max }\right)$.

To estimate the effect of change in upper body exercise on the asymptotic level in torque-time integral within a trial, a quotient $\gamma$ was computed. In trials 1,3 and 5 , parameter $\gamma$ resulted from the division of the mean torque-time integral from 8 to 10 min (mean of 60 samples) by the mean torquetime integral from 4 to $6 \mathrm{~min}$ (also mean of 60 samples) per trial. In trials 2, 4 and 6, parameter $\gamma$ resulted from the division of the mean torque-time integral from 4 to $6 \mathrm{~min}$ by the mean torque-time integral from 8 to $10 \mathrm{~min}$ per trial. Thus, $\gamma>1$ means that the asymptotic level in torque-time integral was higher with upper body exercise than without.

\section{Heart Rate}

The heart rate was estimated by computing the mean rate of every subsequent $10 \mathrm{~s}$ of video registration. The average heart rates between 4 and $6 \mathrm{~min}$ and between 8 and $10 \mathrm{~min}$ after the start of each trial were determined in order to analyse the influ- ence of upper body exercise and the different stimulation and loading conditions on heart rate.

\section{Statistics}

Differences in $M_{\max }, \tau, \xi$ and hcart rates between trials were tested statistically for the pooled data of all subjects using a nonparametric sign test ${ }^{10}$ with a significance level of $\alpha=0.05$. Furthermore, the hypothesis that upper body exercise increases the asymptotic level of the torque-time integral was tested statistically within each trial by testing the hypothesis $\gamma>1$ for the pooled data, again using a non-parametric sign test $(\alpha=0.05)$.

\section{RESULTS}

Figures 3, 4 and 5 exemplify the decay in torquetime integral during all six trials in subject $\mathrm{A}$. The

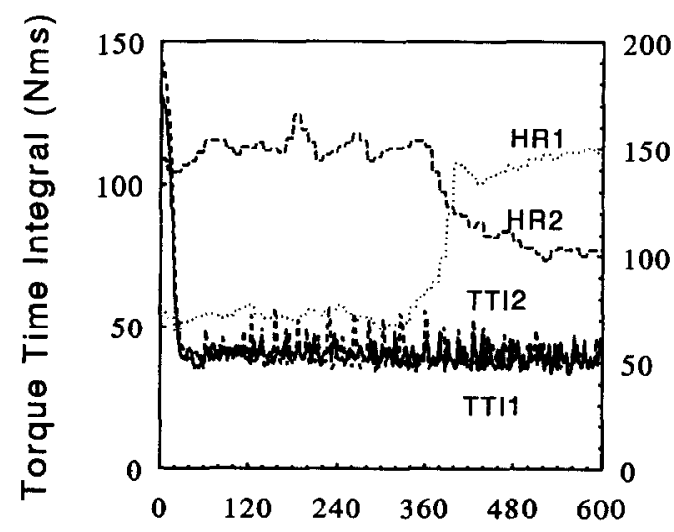

Time (s)

FIG. 3. Decay of torque-time integral during trial 1 (TTI1) and trial 2 (TTL2) for subject A, with isometric loading and continuous stimulation. The corresponding heart rate is also shown (fatigue trial 1: HR1; fatigue trial 2: HR2). The time axis contains the entire duration of the trials, i.e. 300 consecutive stimulation cycles. 


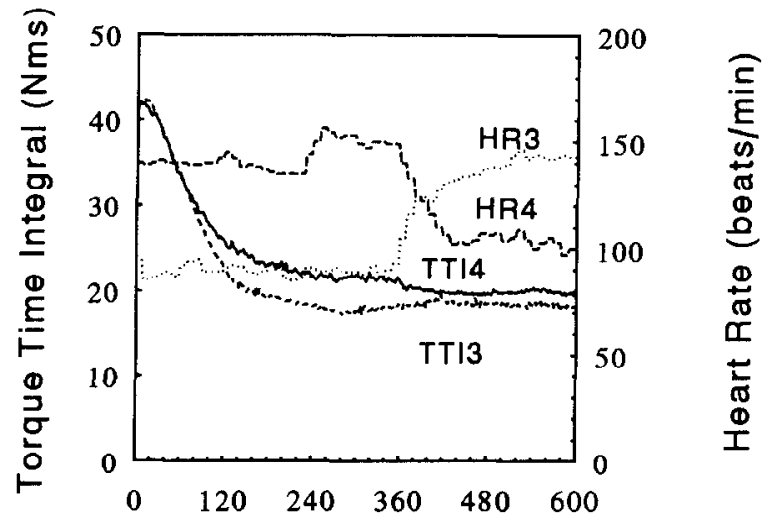

Time (s)

FIG. 4. Decay of torque-time integral during trial 3 (TTI3) and trial 4 (TTI4) for subject A, with isometric loading and intermittent stimulation at a duty cycle of $32 \%$. The corresponding heart rate is also shown (fatigue trial 3 : HR3; fatigue trial 4: HR4). The time axis contains the entire duration of the trials, i.e. 300 consecutive stimulation cycles.

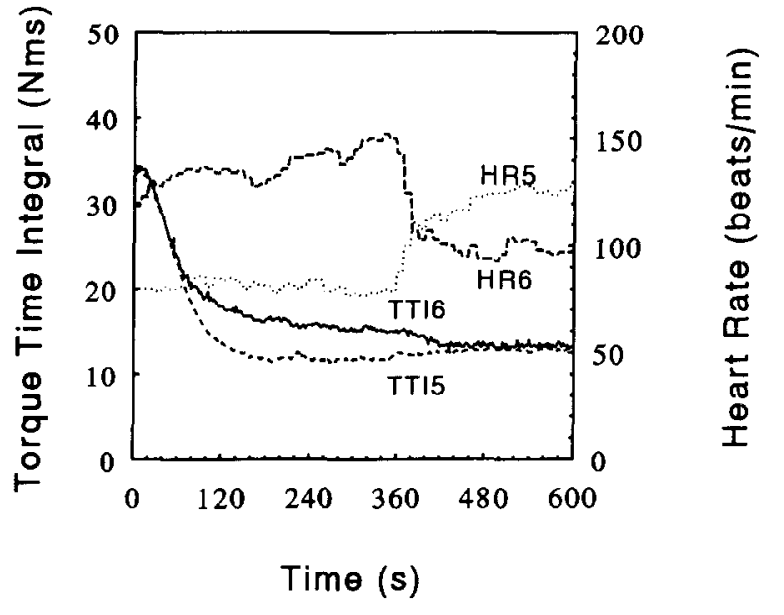

FIG. 5. Decay of torque-time integral during trial 5 (TTI5) and trial 6 (TTI6) for subject $A$, with isokinetic loading and intermittent stimulation at a duty cycle of $32 \%$. The corresponding heart rate is also shown (fatigue trial 5: HR5; fatigue trial 6: HR6). The time axis contains the entire duration of the trials, i.e. 300 consecutive stimulation cycles.

parameterized results for all subjects are given in Tables 3, 4, 5 and 6. Figures 3, 4 and 5 also show the heart rate Upper body exercise significantly increases heart rate. Both onset and offset of upper body exercise give clear responses in heart rate in all subjects.

\section{Initial Maximum of the Torque-Time Integral}

$M_{\max }$ (Table 3) was significantly larger for the isometric trials 1 and 2 with continuous stimulation than for the isometric trials 3 and 4 with intermittent stimulation $(\alpha=0.05)$. This can be explained by the different duty cycles. However, when dividing $M_{\max }$ by the duration of the stimulation bursts no significant difference was found between the continuous stimulation trials 1 and 2 and the intermittent stimulation trials 3 and $4(\alpha=0.05) . M_{\max }$ was significantly larger for the isometric intermittent stimulation trials 3 and 4 than for the isokinetic intermittent stimulation trials 5 and $6(\alpha=0.05)$. This can be explained by the well-known fact that muscle force is lower during concentric than during isometric contractions ${ }^{12,37}$.

\section{Influence of Upper Body Exercise}

The infuence of the upper body exercise on $M_{\max }$, was tested by pairwise comparison of subsequent trials starting with and without upper body exercise. The maximum of the torque-time intregral $M_{\max }$ was significantly smaller in the trials which started with upper body exercise than in the trials without $(\alpha=0.05)$.

\section{Decay Time Constant of Torque-Time Integral}

The decay time constant of the torque-time integral $\tau$ (Table 4) was significantly smaller in trials 1 and 2 compared to trials 3 and $4(\alpha=0.05)$, indicating a faster decline of the output with continuous stimulation (duty cycle $100 \%$ ) than with intermittent stimulation (duty cycle 32\%). This agrees with the findings previously reported by Boom et al. ${ }^{6}$. Furthermorc, $\tau$ was significantly smaller for the isokinetic trials 5 and 6 than for the isometric trials 3 and $4(\alpha=0.05)$. This is in agreement with the results earlier reported by Franken et al. ${ }^{12}$.

\section{Influence of Upper Body Exercise}

The decay time constant of the torque-time integral $\tau$ determined over the first $6 \mathrm{~min}$ of each trial was not found to be significantly influenced by the upper body exercise $(\alpha=0.05)$.

\section{Asymptotic Level in Torque-Time Integral}

The relative asymptote $\xi$ estimated from the first $6 \mathrm{~min}$ of each trial (Table 5) was significantly smaller for the isometric trials 1 and 2 with continu- 
TABLE 3. Initial maximum in the torque-time integral $\left(M_{\text {max }}\right)$ for all trials

\begin{tabular}{lcccccc}
\hline Subject & Trial 1(Nms) & Trial 2(Nms) & Trial 3(Nms) & Trial 4(Nms) & Trial 5(Nms) & Trial 6(Nms) \\
\hline A & 142.9 & 131.4 & 42.6 & 42.1 & 34.2 & 41.2 \\
B & 100.4 & 77.0 & 24.8 & 24.3 & 21.4 & 19.6 \\
C & 179.8 & 149.7 & 47.6 & 41.7 & 29.8 & 26.8 \\
D & 116.5 & 102.7 & 35.3 & 33.7 & 21.2 & 24.4 \\
E & 126.3 & 116.6 & 39.5 & 38.3 & 27.6 & 25.5 \\
\hline
\end{tabular}

$M_{\max }$ was determined by iteratively fitting eqn. (1) to the first $6 \mathrm{~min}$ of the torque-time integral samples of each trial (see Methods: off-line data analysis).

TABLE 4. Time constant of decay in the torque-time integral $(\tau)$ for all trials

\begin{tabular}{lcccccc}
\hline Subject & Trial 1(s) & Trial 2(s) & Trial 3(s) & Trial 4(s) & Trial 5(s) & Trial 6(s) \\
\hline A & 13.5 & 12.7 & 76.7 & 80.2 & 54.0 & 64.3 \\
B & 21.3 & 15.5 & 89.9 & 108.5 & 82.8 & 74.2 \\
C & 17.1 & 16.1 & 60.8 & 70.3 & 38.2 & 36.2 \\
D & 14.4 & 12.8 & 65.3 & 66.2 & 44.7 & 41.2 \\
E & 12.1 & 16.8 & 52.9 & 49.9 & 34.7 & 29.9 \\
\hline
\end{tabular}

The time constant was determined by iteratively fitting eqn. (1) to the first $6 \mathrm{~min}$ of the torque-time integral samples of each trial (see Methods: off-line data analysis).

TABLE 5. Relative asymptote ( $\xi$ ) of the torque-time integral (obtained within $6 \mathrm{~min}$ ) for all trials

\begin{tabular}{lllllll}
\hline Subject & Trial 1 & Trial 2 & Trial 3 & Trial 4 & Trial 5 & Trial 6 \\
\hline A & 0.281 & 0.302 & 0.396 & 0.493 & 0.332 & 0.437 \\
B & 0.276 & 0.331 & 0.480 & 0.533 & 0.341 & 0.406 \\
C & 0.300 & 0.345 & 0.461 & 0.517 & 0.333 & 0.382 \\
D & 0.235 & 0.266 & 0.304 & 0.393 & 0.263 & 0.266 \\
E & 0.254 & 0.249 & 0.346 & 0.364 & 0.245 & 0.277 \\
\hline
\end{tabular}

The relative asymptote was determined by iteratively fitting eqn. (1) to the first $6 \mathrm{~min}$ of the torque-time integral samples of each trial (see Methods: off-line data analysis).

TABLE 6. Descriptive parameter $(\gamma)$ for the change in asymptotic level of the torque-time integral due to upper body exercise within each trial

\begin{tabular}{lcccccc}
\hline Subject & Trial 1 & Trial 2 & Trial 3 & Trial 4 & Trial 5 & Trial 6 \\
\hline A & 0.96 & 1.04 & 1.03 & 1.12 & 1.09 & 1.18 \\
B & 0.91 & 1.07 & 0.93 & 1.17 & 1.02 & 1.19 \\
C & 0.93 & 1.04 & 1.03 & 1.07 & 0.98 & 1.82 \\
D & 1.01 & 1.01 & 1.31 & 1.09 & 1.04 & 1.40 \\
E & 0.87 & 1.01 & 0.97 & 1.30 & 1.04 \\
\hline
\end{tabular}

When $\gamma>1$ the asymptotic level in torque-time integral was higher due to upper body exercise and vice versa.

ous stimulation than for the isometric trials 3 and 4 with intermittent stimulation $(\alpha=0.05)$. This implies that a larger duty cycle results in a smaller relative asymptote. It should be noted that this asymptote is a relative value with respect to $M_{\max }$.
Furthermore, $\xi$ was significantly smaller for the trials 5 and 6 than for the trials 3 and $4(\alpha=0.05)$, supporting the finding that muscles fatigue more during isokinetic than isometric contractions as previously described by Franken et al. ${ }^{12}$. 


\section{Influence of Upper Body Exercise}

Statistical analysis showed that the relative asymptote $\xi$ was significantly higher with upper body exercise (the first $6 \mathrm{~min}$ of trials 2,4 and 5) than without upper body exercise (the first $6 \mathrm{~min}$ of trials 1,3 and 5).

\section{Change in Upper Body Exercise}

Parameter $\gamma$ (Table 6) expresses the influence of upper body exercise on the asymptotic muscle output, assessed from the response to starting or stopping upper body exercise during a trial. Parameter $\gamma$ appeared to be significantly larger than one (all trials pooled; $\alpha=0.05$ ), which means that the asymptote was significantly higher with than without upper body exercise. This is the same conclusion as was drawn from the above inter-trial comparison of relative asymptotes $\xi$ for trials with and without upper body exercise. It should be noted that the actual gain in the asymptotic level due to upper body exercise was variable and on average not very large. The actual responses to starting or stopping upper body exercise during the trials appeared to be marginal in the continuous trials 1 and 2 and more pronounced in the intermittent trials $3,4,5$ and 6 .

\section{Heart Rate}

In all trials, heart rate (Table 7) was markedly larger in the presence of upper body exercise than without. This finding was significant $(\alpha=0.05)$ when comparing the average heart rate between 4 and $6 \mathrm{~min}$ and between 8 and $10 \mathrm{~min}$ within each trial. It was also significant when comparing trials with the same loading and stimulation conditions but different upper body exercise regimes (starting or ending with upper body exercise: trials $2,4,6$ versus $1,3,5)$. No significant differences $(\alpha=0.05)$ were found when comparing the average heart rate between 4 and 6 min of trials with different stimulation and loading conditions but the same upper body exercise regimes.

\section{DISCUSSION}

It can be concluded that upper body exercise augments the asymptotic torque level of electrically stimulated muscles in the paralyzed lower extremities significantly, although no significant effect was found on the rate of torque decay. This significant effect was independently found by comparing trials starting with and withoul upper arm exercise as well as from intra-trial evaluation of the response to starting or stopping upper body exercise. The positive influence of upper body exercise on the output of electrically stimulated muscles in paralyzed extremities may be explained by a bypass of the failing peripheral reflex mechanisms that regulate blood flow to the extremities ${ }^{21,23}$ and the muscle pump effect, which may enhance cardiac output ${ }^{9,11,17,18}$. This experimental finding is in accordance with the results of Glaser et al. ${ }^{17}$, who evaluated knee extension angle during weight lifting with and without maximal voluntary upper body effort. They found a significant and pronounced reduction of fatigue of the stimulated muscles. The significant effect found in the current study is not as pronounced, and sometimes even slightly reversed. This may be caused by the lower level of upper body exercise.

Our primary interest is the effect of upper body effort in FNS-assisted mobility in paraplegic subjects. This upper body effort is an essential prerequisite in current FNS mobility systems for paraplegics ${ }^{1,15,24,26,29}$. It is required for balancing the body and supplementing the output of the electrically stimulated muscles, which may be insufficient to perform tasks such as standing up. Our experi-

TABLE 7. Average heart rate between 4 and 6 min and between 8 and 10 min after the start of the trials

\begin{tabular}{lllllll}
\hline & \multicolumn{5}{c}{ Heart rate (beats/min) } \\
\hline Subject & Trial 1 & Trial 2 & Trial 3 & Trial 4 & Trial 5 \\
\hline A & $72 / 146$ & $151 / 103$ & $90 / 142$ & $151 / 104$ & $80 / 126$ & $155 / 98$ \\
B & $90 / 112$ & $129 / 99$ & $101 / 134$ & $146 / 120$ & $108 / 136$ & $137 / 119$ \\
C & $75 / 114$ & $119 / 83$ & $80 / 134$ & $156 / 108$ & $94 / 142$ & $153 / 105$ \\
D & $81 / 107$ & $102 / 78$ & $87 / 119$ & $120 / 94$ & $77 / 110$ & $108 / 81$ \\
E & $66 / 120$ & $113 / 62$ & $65 / 106$ & $103 / 58$ & $46 / 92$ & $105 / 53$ \\
\hline
\end{tabular}

The first number for each trial gives the average heart rate between 4 and $6 \mathrm{~min}$, the second between 8 and $10 \mathrm{~min}$. 
mental results indicate that the effect of muscle fatigue in paralysed muscles which are electrically stimulated may be reduced by upper body effort. Therefore, upper body effort in FNS mobility systems for paraplegics should not merely be considered as an inevitable requirement, but as a prerequisite for effective FNS support of mobility in part of the paraplegic population. However, it should be noted that the effect of upper body effort on the fatigue of stimulated paralysed muscles may be different for walking than for sitting, which is the condition under which the current experiments were performed. The predicted effects during walking still need to be verified.

Several other studies report on the combination of arm exercise and electrically stimulated lower extremity muscles in SCI subjects (for a review see Connor ${ }^{8}$ ). The main attention in these studies was on the facilitating influence of electrical stimulation on the cardiovascular and respiratory function of $\mathrm{SCl}$ individuals. Glaser et al. ${ }^{17}$ reported an increased oxygen uptake and pulmonary ventilation when combining FNS with voluntary upper body exercise, which has important consequences for the physical training of SCI subjects.

The influences of duty cycle and loading on muscle fatigue were consistent with previous studies: in accordance with the findings reported by Boom et al. ${ }^{6}$, continuous stimulation (duty cycle 100\%) resulted in a faster decay of the muscle output than intermittent stimulation (duty cycle 32\%). Furthermore, isokinetic loading resulted in a faster decline and a lower relative asymptote of muscle output than isometric loading, as was earlier reported by Franken et al. ${ }^{12}$. In this analysis the asymptote was considered relative to the maximum output (torquetime integral of a cycle). This maximum output, occurring at the beginning of the trials, was smaller for isokinetic loading than for isometric loading. This is also consistent with earlier findings ${ }^{12,37}$ and can be explained by the hyperbolic Hill relation, which describes the muscle force as a function of contraction velocity.

As indicated in Table 1, the quadriceps muscles of all subjects were found to be normally excitable. However, in subject $\mathrm{B}$, minor peripheral damage to the innervation of parts of the quadriceps cannot be excluded because of the relatively low injury level (T11/12). No clear indications for such damage were found in the response of the muscles. Although subject $B$ showed the lowest maximal torque-time integrals (Table 3 ), the values are not outliers when considering the variance in maximal torque-time integrals over the five subjects.

In the experiments, the arbitrary choice was made to stimulate left leg quadriceps in all subjects, and not the quadriceps of the dominant side. It should be noted that the differences in performance between right and left sides in these paraplegic individuals may be determined more by differences in training and spasm than dominant use of either side before the date of injury.

On the basis of our results, an intra-subject variability analysis could not be made because each trial was performed only once for each subject. However, the experiment was repeated in a sufficient number of subjects to yield statistically significant results on an inter-subject level.

In the design of the protocol, care was taken that subsequent trials would influence each other only minimally. For this reason, the experiment started with a warm-up session and $50 \mathrm{~min}$ of rest was allowed between trials. However, it cannot be excluded that the muscle did not fully recover to the same initial level as previous trials, which may have a systematic influence on the results. Such a possible systematic effect makes conclusions about increased performance in later trials more solid, but a conclusion about decreased performance questionable. The parameter most sensitive for possible systematic reduction of the muscle output in later trials is the maximum torque-time integral per cycle $M_{\max }$. However, no evidence of such an effect in this parameter was found. For example, when comparing the continuous stimulation trials 1 and 2 with the intermittent stimulation trials 3 and $4, M_{\max }$ was significantly larger in trials 1 and 2 than in trials 3 and 4 due to the difference in duty cycle. When dividing $M_{\max }$ by the duration of the stimulation bursts, no significant difference remained $(\alpha=0.05)$. However, it cannot be excluded that the order of trials influenced our finding that $M_{\max }$ was significantly smaller in the trials which started with upper body exercise than in the trials without. For several reasons, our conclusion that the asymptotic torque time integral increases in the presence of upper body effort is firm. First, the relative asymptotic torque-time integral $\xi$ was found to increase in later trials. Second, the effect of upper body exercise was also determined within trials by analysing the response to starting or stopping upper body exercise during the trials. These intra-trial evaluations (parameter $\gamma$ ) showed the same effect of upper body exercise as the comparison between trials. They 
even led to the conclusion that the asymptotic level of torque-time integral per cycle increases not only relative to the initial maximum, but also in absolute value.

\section{CONCLUSIONS}

1. Upper body exercise yielded a significant increase in the asymptotic level of knee torque generated by stimulation of the quadriceps in SCI subjects. The actual change of the asymptotic level was variable between subjects and marginal during continuous stimulation.

2. No significant influence of upper body exercise on the rate of torque decline was found.

3. Upper body exercise significantly increased heart rate.

4. Continuous and intermittent stimulation did not yield significant differences in heart rates under the same upper body exercise conditions.

5. Isometric and isokinetic loading did not yield significant differences in heart rates under the same upper body exercise conditions.

6. Knee torque declined faster and to a lower relative asymptote during continuous stimulation than during intermittent stimulation.

7. Knee torque declined faster and to a lower relative asymptote during isokinetic concentric loading than during isometric loading.

Acknowledgements: We wish to thank the subjects for their cooperation and acknowledge that the experiments were performed in the laboratory of the Center for Rehabilitation Technology at the rehabilitation centre 't Roessingh (Enschede, The Netherlands).

\section{REFERENCES}

1. Andrews BJ, Barnett RW, Phillips GF, Kirkwood CA: Rulebased control of a hybrid FES orthosis for assisting paraplegic locomotion. Automedica 11:175-199, 1989.

2. Astrand PO, Rodahl K: Textbook of Work Physiology, Physiological Bases of Exercise, McGraw-Hill, 1986.

3. Baratta R, Ichie M, Hwang SK, Solomonow M: Orderly stimulation of skeletal muscle motor units with tripolar nerve cuff electrode. IEEE Trans Biomed Engng 36:836-843, 1989.

4. Bevan L, Laouris Y, Reinking RM, Stuart DG: The effect of the stimulation pattern on the fatigue of single motor units in adult cats. J Physiol 449:85-108, 1992.

5. Binder-Macleod ST., Barker CB: Use of a catchlike property of human skeletal muscle to reduce fatiguc. Muscle Nerve 14:850-857, 1991.

6. Boom HBK, Mulder AJ, Veltink PH: Fatigue during functional neuromuscular stimulation. Progr Brain Res 97:409$418,1993$.

7. Carroll SG, Triolo RJ, Chizeck HJ, Kobetic R, Marsolais
EB: Tetanic response of electrically stimulated paralyzed muscle at varying interpulse interval. IEEE Trans Biomed Engng 36:644-653, 1989.

8. Connor FJ: Cardiorespiratory responses to exercise and training by persons with spinal cord injuries: a review. Clin Kinesiol 00:13-17, 1991.

9. Davis GM, Servedio FJ, Glaser RM, Gupta SC, Suryaprasad AG: Cardiovascular responses to arm cranking and FNSinduced leg exercise in paraplegics. $J$ Appl Physiol 69:671$677,1990$.

10. Devors JL: Probability and Statistics for Engineering and Sciences, CA Brooks/Cole, Monterey, 1987.

11. Flandrois R, Grandmontagne M, Gerin H, Mayet $M H$, Jehl JL, Eyssette M: Aerobic performance in paraplegic subjects. Eur J Appl Physiol 55:604-609, 1986.

12. Franken HM, Veltink PH, Fidder M, Boom HBK: Fatigue of intermittently stimulated human quadriceps during imposed cyclical lower leg movements. Int J Electromyogr Kinesiol $3: 3-12,1993$

13. Franken HM, Veltink PH, Van Harn A, Boom HBK: The influence of a stimulation pattern on force and fatigue profiles in FES-induced quadriceps contractions in paraplegics. Basic Appl Myol 4:173-186, 1994.

14. Franken HM, Veltink PH, Baardman G, Redmeijer RA, Boom HBK: Cycle-to-cycle control of the swing phase of paraplegic gait induced by surface electrical stimulation. Med Biol Engng Comput 33:440-451, 1995.

15. Franken HM, Veltink PH, Boom HBK: Restoring gait in paraplegics by functional electrical stimulation. IEEE Engng Med Biol Mag 13:564-570, 1994.

16. Giat Y, Mizrahi J, Levy M: A musculoskeletal model of the fatigue profiles of paralyzed quadriceps muscle under FES. IEEE Trans Biomed Engng 40:664-674, 1993.

17. Glaser Rl 1, Strayer JR, May KP: Combined FES leg and Voluntary Arm Excercise of SCI Patients. In: Proceedings of the IEEE EMBS 7th Congress, pp 308-313, 1989.

18. Glaser RM: Physiological aspects of spinal cord injury and functional neuromuscular stimulation. Cent Nerv Syst Trauma $3: 49-62,1986$.

19. Gurfinkel VS, Levik YU: Dependence of contraction of the muscle on the sequence of stimulating pulses. Biophysics 18:121-127, 1973.

20. Gurfinkel VS, Levik YU: Effects of a doublet or omission and their connexion with the dynamics of the active state of human muscles. Biophysics 19:925-931, 1974.

21. Hopman MTE, Oeseburg B, Binkhorst RA: Cardiovascular responses in paraplegic subjects during arm cxcreisc. Eur $J$ Appl Physiol 65:73-78, 1992.

22. Hopman MTE, Oeseburg B, Binkhorst RA: The effect of an anti-G suit on cardiovascular responses to exercise in persuns with paraplegic. Med Sci Sports Exerc 24:984-990, 1992.

23. Hopman MTE, Van Asten WNJC, Oeseburg B: Blood flow changes below and above the spinal cord lesion during arm exercise in individuals with paraplegic. Appl Physiol (Suppl) 69:S26, 1994.

24. Isakov E, Mizrahi J, Najenson T: Biomechanical and physiological evaluation of FES-activated paraplegic patients. $J$ Rehab Res Dev 23:9-19, 1986

25. Karu ZZ, Durfee WK: Using pulse doublets to enhance muscle force for FES. In: 14th International Conference IEEE/FMRS, ed by Morucci JP, Plonsey R. Coatrieux JL, Laxminarayan S, Paris, 1992, pp 1356-1357.

26. Kralj A, Bajd T, Turk R, Krajnik J, Benko H: Gait restoration in paraplegic patients: a feasibility demonstation using multichannel surface electrode FES. J Rehab Res Dev 20:3$20,1983$.

27. Kralj A, Bajd T, Turk R, Benko H: Posture switching for prolonging functional electrical stimulation standing in paraplegic patients. Paraplegia 24:221-230, 1986. 
28. Levy M, Mizrahi J, Susak Z: Recruitment, force and fatigue characteristics of quadriceps muscles of paraplegics isometrically activated by surface functional electrical stimulation. $J$ Biomed Engng 12:150-156, 1990.

29. Marsolais EB, Kobetic R: Functional electrical stimulation for walking in paraplegic. J Bone Joint Surg 69A:728$733,1987$.

30. Mitchell JH: Neural control of the circulation during exercise. Med Sci Sports Exerc 22:141-154, 1990.

31. Peckham PH: Functional electrical stimulation: current status and future prospects of applications to the neuromuscular system in spinal cord injury. Paraplegia 25:279-288, 1989.

32. Petrofsky JS, Phillips CA: The influence of recruitment order and fibre composition on the force-velocity relation and fatiguability of skeletal muscles in the cat. Med Biol Fngng Comput 18:381-390, 1980.

33. Rowell LB, O'Leary DS: Reflex control of the circulation during exercise: chemoreflexes and mechanoreflexes. J Appl Physiol 69:407-418, 1990.

34. Salmons S, Hendriksson J: The adaptive response of skeletal muscle to increased use. Muscle Nerve 4:94 105, 1981.
35. Stein RB, Parmiggiani FL: Optimal motor patterns for activating mammalian muscle. Brain Res 175:372-376, 1979.

36. Solomonow M, Baratta R, Hirokawa S, Rightor N, Walker W, Beaudette P, Shoji H, D'Ambrosia R: The RGO generation II: muscle stimulation powered orthosis as a practical walking system for thoracic paraplegics. Orthopaedics 12:1309_ 1315, 1989.

37. Triolo RJ, Robinson DE, Betz RR: Force-velocity and lengthtension properties of stimulated human quadriceps in spinal cord injured children. In: Proceedings of the IEEE EMBS 1/th International Congress, pp 967-968, 1989.

38. Veltink PII: Control of FES-induced cyclical leg movements of the lower leg. Med Biol Engng Comput 29:NS8-NS12, 1991.

39. Zajac FE, Young JL: Properties of stimulus trains producing maximum tension-time area per pulse from single motor units in medial gastrocnemius muscle of the cat. $J$ Neurophysiol 43:1206 -1220, 1980 . 